\title{
TAZ overexpression is associated with epithelial-mesenchymal transition in cisplatin-resistant gastric cancer cells
}

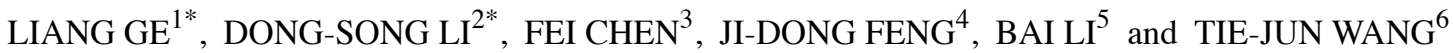 \\ Departments of ${ }^{1}$ Anesthesiology, ${ }^{2}$ Surgery, The First Hospital of Jilin University; \\ ${ }^{3}$ Abdominal Tumor Medical Department, Jilin Provincial Tumor Hospital; ${ }^{4}$ Department of Normal Surgery, \\ Jilin Province Hospital of Traditional Chinese Medicine; Departments of ${ }^{5}$ Colorectal and Anal Surgery, \\ ${ }^{6}$ Orthopedic Traumatology, The First Hospital of Jilin University, Changchun, Jilin 130021, P.R. China
}

Received February 14, 2017; Accepted April 13, 2017

DOI: $10.3892 /$ ijo.2017.3998

\begin{abstract}
Gastric cancer is one of the common malignant diseases. The poor treatment outcome is mainly due to chemotherapeutic resistance. Therefore, it is important to determine the molecular mechanism of drug resistance in gastric cancer. To explore the mechanisms of cisplatin resistance in gastric cancer cells, several approaches were performed including MTT assay, real-time RT-PCR, western blot analysis, migration and invasion assays, wound healing assay, and transfection. We found that cisplatin-resistant (CR) gastric cancer cells acquired epithelial-mesenchymal transition (EMT) phenotype. The CR cells with EMT features obtained higher migratory and invasive activities. Moreover, we observed that TAZ was highly expressed in CR cells. Consistently, depletion of TAZ caused partial reversal of EMT to MET in CR cells. Our results suggest that TAZ plays a pivotal role in CR-induced EMT. Targeting TAZ could be a potential therapeutic strategy for gastric cancer.
\end{abstract}

\section{Introduction}

Gastric cancer is one of the most common malignancies in the United States. There is a decline in gastric cancer occurrence partly due to a lower prevalence of Helicobacter pylori infection because of improved hygiene and a higher consumption of vegetables and lower salt intake (1). However,

Correspondence to: Dr Bai Li, Department of Colorectal and Ana Surgery, The First Hospital of Jilin University, Changchun, Jilin 130021, P.R. China

E-mail: bailichangchun@126.com

Dr Tie-Jun Wang, Department of Orthopedic Traumatology, The First Hospital of Jilin University, Changchun, Jilin 130021, P.R. China E-mail: tiejunwangjilin@163.com

${ }^{*}$ Contribute equally

Key words: gastric cancer, chemoresistance, cisplatin, TAZ, epithelial-mesenchymal transition it is estimated that 28,000 new cases and 10,960 deaths will occur in 2017 in the US (1). In China 679,100 new gastric cancer cases occurred in 2015 , being one of the four most common cancers diagnosed (2). Notably, gastric cancer is the second leading cause of cancer death worldwide. Current therapies in clinic mainly include surgery, chemotherapy, and chemoradiation for gastric cancer (3). Although surgery remains the curative therapy, chemotherapy is the important treatment for gastric cancer (4). Due to intrinsic or acquired resistance to chemotherapeutic drug, chemotherapy often fail to achieve effective treatment benefit for gastric cancer patients. Therefore, it is important to determine the molecular mechanism of drug resistance in gastric cancer (5).

Cisplatin has been known as an effective chemotherapeutic drug for the treatment of human malignancies including gastric cancer (6). Cisplatin and fluoropyrimidine-based chemotherapy and trastuzumab have been widely used for advanced stage patients with epidermal growth factor 2 (EGFR2) positivity (7). Unfortunately, cisplatin resistance often occurred during chemotherapeutic treatment (8). Emerging evidence has suggested that drug resistant tumor cells acquired epithelial-mesenchymal transition (EMT) (9). During EMT progress, epithelial cells transit to mesenchymal phenotype, leading to loss of epithelial cell-cell junction and actin cytoskeleton reorganization (10). Subsequently, the expression of epithelial marker E-cadherin was downregulated, but the expression of mesenchymal markers was upregulated, including Vimentin, Snail, Slug, ZEB1 (zinc-finger E-box binding homeobox 1), and ZEB2 (11). A study revealed that chemoresistance to cisplatin induced EMT in human lung adenocarcinoma cells (12). Similarly, another study identified that EMT is associated with cisplatin resistance in gastric cancer cells (13). Furthermore, EMT transcription factor Snail and Slug directly contribute to cisplatin resistance in ovarian cancer cells (14). Although these studies indicated the cisplatin resistance molecular basis, the precise mechanisms of cisplatin resistance are still elusive.

TAZ (transcriptional co-activator with PDZ-binding motif) has been characterized to be involved in gastric tumorigenesis (15). It is known that YAP1 (Yes-associated protein 1) and its paralog TAZ are key factors which are negatively regulated by the Hippo pathway (16). TAZ exerts 
its oncogenic function through interaction with TEAD transcription factors. Notably, TAZ was highly expressed in gastric cancer samples (17). Strikingly, the expression of TAZ had a close correlation with lymphatic metastasis and tumor TNM (tumor, node, metastasis) stage in gastric cancer (18). Several studies have revealed that TAZ was critically involved in drug resistance in a variety of human cancers $(19,20)$. For instance, taxol resistance is mediated by TAZ and its downstream transcriptional targets Cyr61 and CTGF (connective tissue growth factor) in breast cancer cells (21). Moreover, TAZ promoted EMT and cancer stem cell maintenance in oral cancer (22). Due to TAZ playing a key role in drug resistance and EMT, it is important to determine whether TAZ could regulate drug resistance and EMT in gastric cancer. In the present study, we investigated whether cisplatin-resistant (CR) cells exhibit EMT features in gastric cancer. We also elucidate the role of TAZ in CR-mediated EMT in gastric cancer.

\section{Materials and methods}

Reagents. MTT [3-(4,5-dimethythiazol- 2-yl)-2,5-diphenyl tetrazolium bromide] was obtained from Sigma (St. Louis, MO, USA). Primary antibodies including anti-TAZ, antiVimentin, anti-Snail, anti-Slug, anti-ZO1, anti-E-cadherin, anti- $\beta$-catenin, and anti-Tubulin were purchased from Santa Cruz Biotechnology (Santa Cruz, CA, USA). Lipofectamine 2000 and (Roswell Park Memorial Institute) RPMI-1640 medium were purchased from Invitrogen (Carlsbad, CA, USA). The non-specific control siRNA and TAZ siRNA oligonucleotides were purchased from GenePharma (Shanghai, China). Tumor Invasion Assay System was obtained from BD Biosciences (Bedford, MA, USA). Protein assay kit was purchased from Bio-Rad Laboratories (Hercules, CA, USA).

Cell culture. Human gastric cancer cell lines MGC803 and SGC7901 were cultured in RPMI-1640 medium supplemented with 5\% FBS (fetal bovine serum), penicillin, and streptomycin. Cells were maintained in a humidified $5 \%$ $\mathrm{CO}_{2}$ incubator at $37^{\circ} \mathrm{C}$. Cells were cultured in medium with increasing concentrations of cisplatin for more than 6 months to establish CR cell lines (23).

MTT assay. The gastric cancer cells were seeded in each well of the 96-well plates. After $24 \mathrm{~h}$ incubation, the cells were treated with different concentrations of cisplatin for $72 \mathrm{~h}$. MTT assay was conducted as previously described (24).

Transwell invasion assay. Cell invasion was detected using 24 -well inserts with matrigel in $8-\mu \mathrm{m}$ pores as previously described (24). The invasive activity of cells was detected by using Transwell Invasion kit following the protocol from the manufacturer as previously described (24). Briefly, cells were cultured in the upper chamber of the inserts and RPMI-1600 medium with 10\% FBS was added in the lower chamber. After $12 \mathrm{~h}$ at $37^{\circ} \mathrm{C}$, cells on the upper side of the Transwell were removed. The invading cells on the lower side were fixed and stained with Giemsa solution as well as photographed under a microscope.
Cell attachment and detachment assay. Cell attachment and detachment assays were performed as previously described (25). Briefly, for attachment assay, gastric cancer cells were added in 24-well plates. After $1 \mathrm{~h}$ of incubation, we removed the unattached cells and counted the attached cells. For cell detachment assay, after $24 \mathrm{~h}$ incubation, the detached cells by $0.05 \%$ trypsin digestion for 2 min were counted. The remaining attached cells were trypsinized and counted. The results are presented as a percentage of the attached or detached cells to total cells.

Wound healing assay. The cells were seeded in 6-well plate. After the cells reached $>90 \%$ confluency, the scratch wound was generated by a pipette tip. After $16 \mathrm{~h}$, photographic images were taken (26).

Reverse transcription-PCR (polymerase chain reaction) analysis for gene expression. The total RNA was isolated with TRIzol (Invitrogen) according to the manufacturer's protocols. The real-time PCR reactions and the primers used in PCR reaction were previously described (24).

Western blot analysis: Cells were lysed with RIPA buffer supplemented with protease inhibitors. The protein concentrations were measured by the Bio-Rad protein assay kit. The same amount of protein samples were separated by SDS-PAGE (sodium dodecyl sulfate polyacrylamide gel electrophoresis) and then electrotransferred to the membranes. The membranes were immunoblotted with primary antibodies for western blotting as previously described (27).

Transfection. Gastric cells were seeded in 6-well plates and transfected with TAZ siRNA, or control siRNA (GenePharma) using Lipofectamine 2000 as previously described (24). After the transfection, the cells were applied for further investigation as described under the results section.

Statistical analysis. Statistical analyses were performed to evaluate significance between different groups by GraphPad Prism 5.0 (Graph pad Software, La Jolla, CA, USA). All data were expressed as mean $\pm \mathrm{SD}$ (standard deviation). $\mathrm{P}<0.05$ was considered to indicate a statistical significant difference.

\section{Results}

Establishment of cisplatin-resistant gastric cancer cell lines. To determine the acquired function of drug resistant gastric cancer cells, the cisplatin-resistant (CR) gastric cancer cells were established. MGC803 and SGC7901 cells were exposed to low concentration of cisplatin for several days. The dead cells were removed and increasing concentrations of cisplatin were added in culture medium. After gastric cancer cells were cultured for more than 6 months with increasing concentrations of cisplatin, CR cells (MGC803/DDP and SGC7901/DDP) cells were established (23). Our MTT assay showed that $3 \mu \mathrm{M}$ cisplatin led to approximately $50 \%$ cell growth inhibition in both MGC803 and SGC7901 cells (Fig. 1A). Moreover, $4 \mu \mathrm{M}$ cisplatin treatment caused more than $60 \%$ and $80 \%$ growth inhibition in MGC803 and SGC7901 cells, respectively (Fig. 1A). However, $2 \mu \mathrm{M}$ cisplatin did not inhibit cell growth in 
A

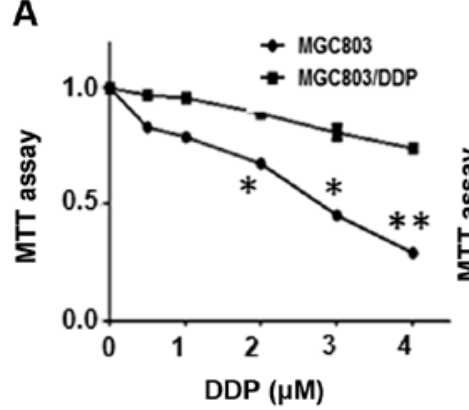

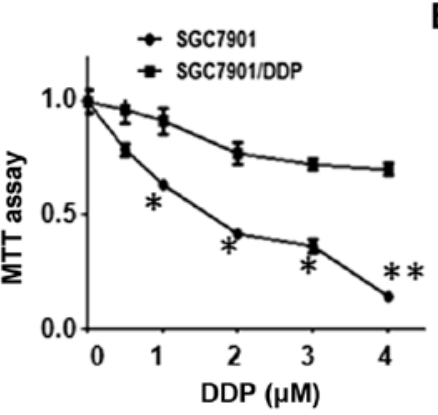

B

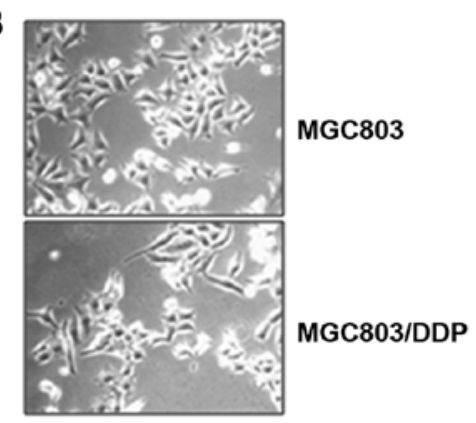

C

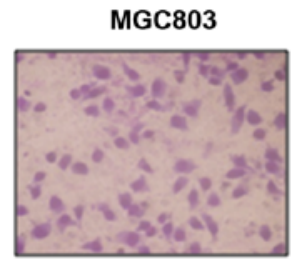

SGC7901

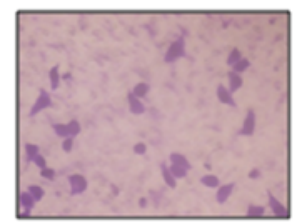

D

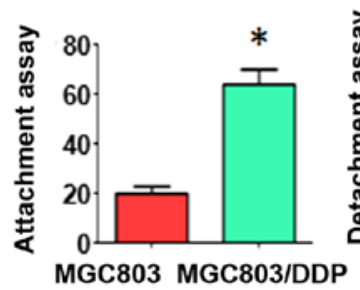

MGC803/DDP

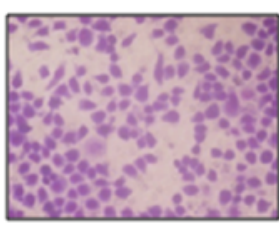

SGC7901/DDP
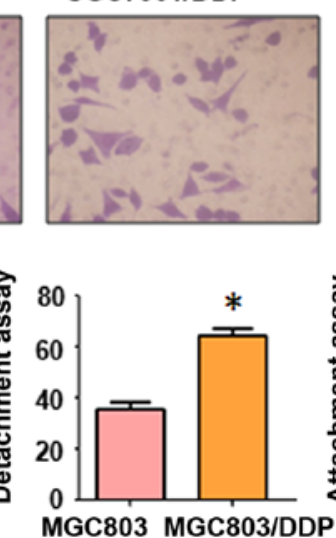
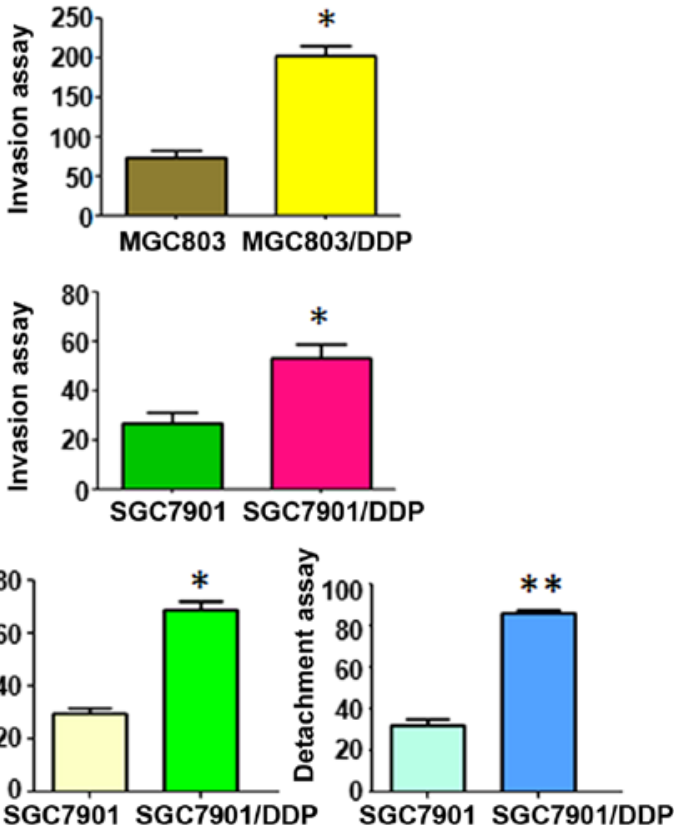

Figure 1. Cisplatin-resistant (CR) cells exhibited EMT phenotype. (A) MTT assay was conducted in parental and CR gastric cancer cells. ${ }^{*} \mathrm{P}<0.05,{ }^{* * *} \mathrm{P}<0.01$ vs. control. (B) Cell morphology was observed by microscopy in parental and CR cells. Parental MGC803 and SGC7901 cells displayed an epithelioid appearance, whereas their CR cells showed elongated, irregular fibroblastoid morphology. (C) Invasion assay was performed to measure the invasive capacity in parental and CR cells. ${ }^{\mathrm{P}}<0.05$ vs. control. (D) Cell attachment and attachment assays were assessed in parental and CR cells. " $\mathrm{P}<0.05,{ }^{* *} \mathrm{P}<0.01$ vs. control.

both CR cells (Fig. 1A). The resistant cells were continuously cultured in medium with $2 \mu \mathrm{M}$ cisplatin for the following study.

CR cells exhibit EMT features. It has been documented that drug resistant cells acquired EMT phenotype (28,29). In line with this concept, MGC803/DDP and SGC7901/DDP cells displayed morphologic changes, such as EMT phenotype. Both MGC803/DDP and SGC7901/DDP cells become elongated, and fibroblastoid in morphology, whereas MGC803 and SGC7901 were a rounded shape (Fig. 1B). EMT-type cells often have aggressive characteristics. Our Transwell invasion assay showed that the numbers of invasive cells were increased in CR cells compared with parental cells (Fig. 1C). Consistently, CR cells exhibited enhanced capacity of detachment and attachment (Fig. 1D). Similarly, our wound healing assay results demonstrated that CR cells acquired increased motility activity (Fig. 2A). Altogether, CR cells acquired EMT characteristics.

CR cells have EMT molecular marker changes. To further confirm whether CR cells acquired EMT, we detected the expression of EMT molecular markers in CR cells and their parental cells, including N-cadherin, E-cadherin, Vimentin, Slug, and ZO-1. Our real-time RT-PCR results indicated that the expression of E-cadherin and ZO-1 was downregulated in CR cells, whereas the levels of N-cadherin, Vimentin, and Slug were upregulated in CR cells (Fig. 2B). Importantly, our western blotting data validated that epithelial molecules were decreased in CR cells, but mesenchymal markers were increased in CR cells (Fig. 3). These findings suggest that $\mathrm{CR}$ cells acquired a mesenchymal feature.

Overexpression of TAZ in CR cells. Several studies have revealed that TAZ is associated with EMT in cancers $(30,31)$. To determine whether TAZ was involved in CR-induced EMT process, real-time RT-PCR and western blotting were performed to measure the transcription and translation levels of TAZ in CR cells. We observed that both mRNA and protein levels of TAZ were significantly upregulated in CR cells (Fig. 4A). Notably, Notch1, a target of TAZ, was also increased (Fig. 4A). We also found that $\beta$-catenin is highly expressed in CR cells (Fig. 4A). Therefore, our results displayed that the acquisition of EMT might be due to higher expression of TAZ in CR cells. 
A
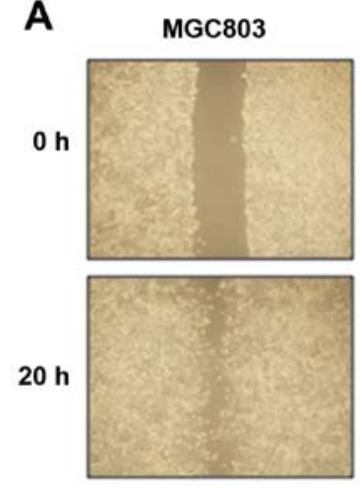

MGC803/DDP
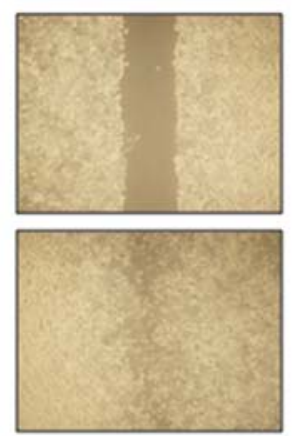

SGC7901

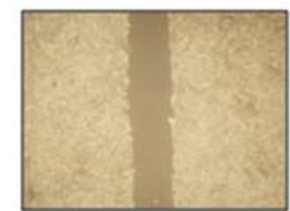

$20 \mathrm{~h}$

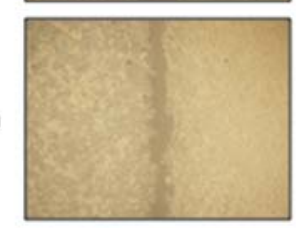

SGC7901/DDP
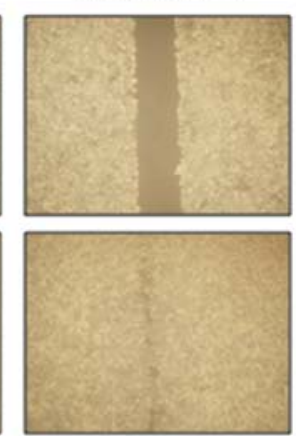

B
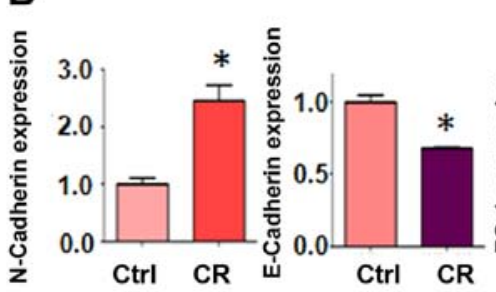

SGC7901
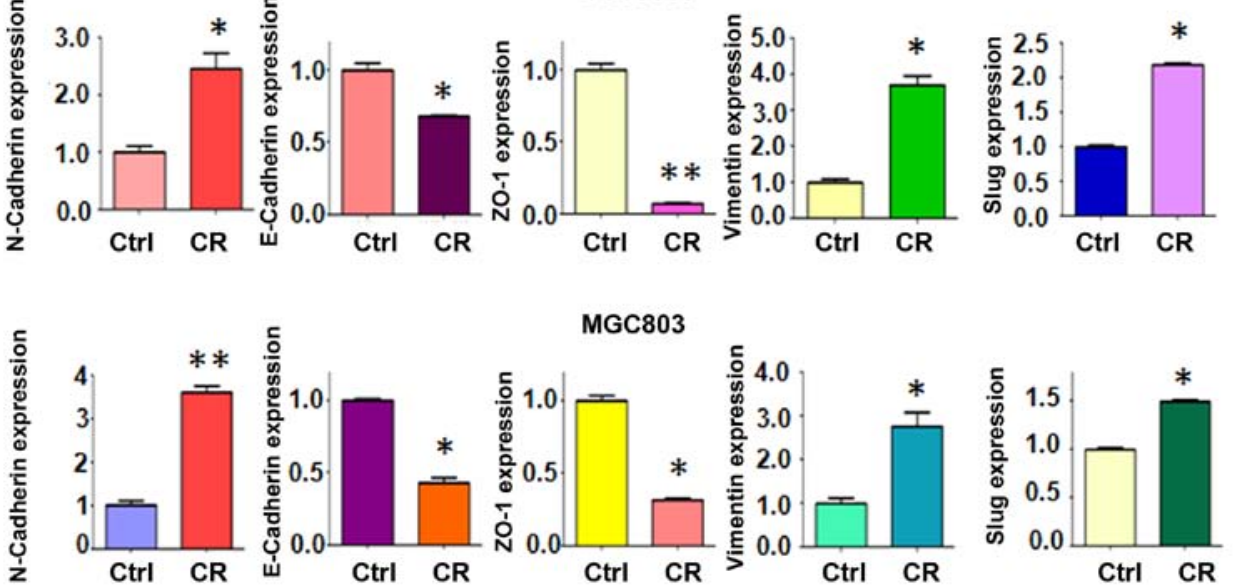

Figure 2. Cisplatin-resistant (CR) cells have enhanced motility activity. (A) Wound assays were performed in parental and CR cells. (B) Real-time RT-PCR assay was conducted to detect the expression of EMT markers in parental and CR cells. ${ }^{\mathrm{P}}<0.05 \mathrm{CR},{ }^{* *} \mathrm{P}<0.01$ vs. control. Ctrl, control.

Depletion of TAZ reverses EMT to MET in CR cells. To further dissect the role of TAZ in CR-triggered EMT, we knocked down the expression of TAZ in CR cells. Our TAZ siRNAs significantly downregulated the expression of TAZ in CR cells (Fig. 4B). We selected TAZ siRNA3 for the following study. We found that depletion of TAZ reverses EMT to mesenchymalepithelial transition (MET) in CR cells (Fig. 4C). Noteworthy, downregulation of TAZ decreased cell attachment and detachment activities (Fig. 4D). These data suggest that TAZ plays an essential role in CR-induced EMT.

Depletion of TAZ retards motility and invasion in CR cells. To deeper define the function of TAZ in CR cells, Transwell invasion assay was conducted. Our invasion results displayed that downregulation of TAZ significantly inhibited cell invasion in CR cells (Fig. 5A). In support of this, our wound healing assay showed that depletion of TAZ reduced cell motility in CR cells (Fig. 5B). Therefore, TAZ plays an important role in regulation of cell migration and invasion in CR cells.

Depletion of TAZ regulates expression of EMT markers. Next, we explore whether knockdown of TAZ could regulate the expression of EMT markers. To achieve this goal, the mRNA and protein levels of EMT markers were measured by realtime RT-PCR and western blotting, respectively. We found that downregulation of TAZ increased the expression of epithelial markers, but decreased the level of mesenchymal markers
(Figs. 5C and 6A and B). Therefore, these results further confirmed the role of TAZ in regulation of EMT in CR cells.

Downregulation of TAZ enhances CR cells to cisplatin sensitivity. To determine whether depletion of TAZ expression enhances CR cells to cisplatin sensitivity, MTT assay was used to detect the cell growth in TAZ siRNA transfected CR cells. We observed that downregulation of TAZ significantly attenuated cell growth inhibition approximately $20-30 \%$ induced by $2 \mu \mathrm{M}$ cisplatin (Fig. 6C). These MTT results implied that downregulation of TAZ could enhance CR cells to cisplatininduced cell growth inhibition.

\section{Discussion}

Gastric cancer is the fourth most common cancer worldwide. Since chemotherapy often has treatment failure due to drug resistance, it is pivotal to explore the mechanisms of chemotherapy resistance in gastric cancer. It is clear that reasons of drug resistance are complex and multifactorial. Thus, it is necessary to define the mechanisms of chemoresistance in gastric cancer (23). In the present study, we aimed to elucidate the molecular basis of chemoresistance in gastric cancer. We observed that cisplatin-resistant (CR) gastric cancer cells obtained EMT features, leading to enhanced motility and invasion. Mechanistically, higher expression of TAZ was found in CR cells. Moreover, depletion of TAZ reversed CR-induced 

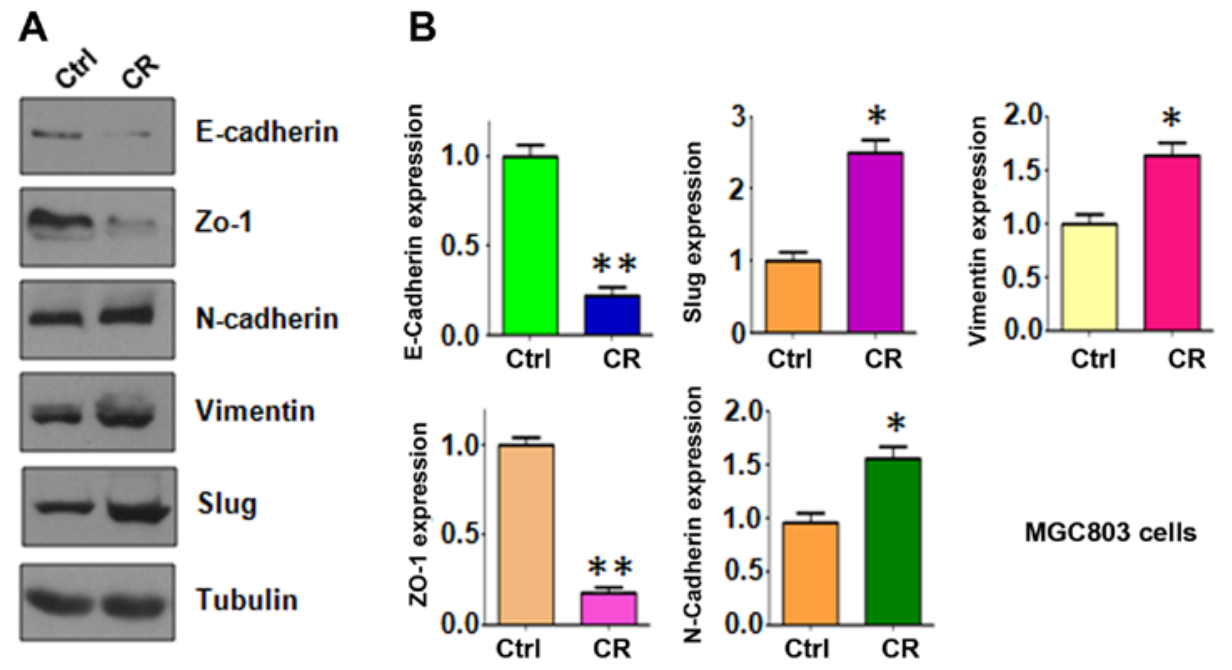

MGC803 cells

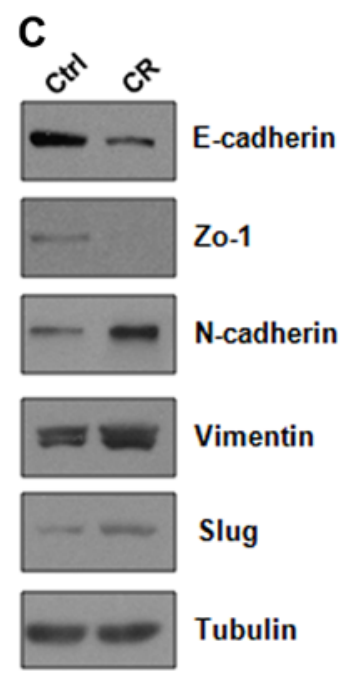

D
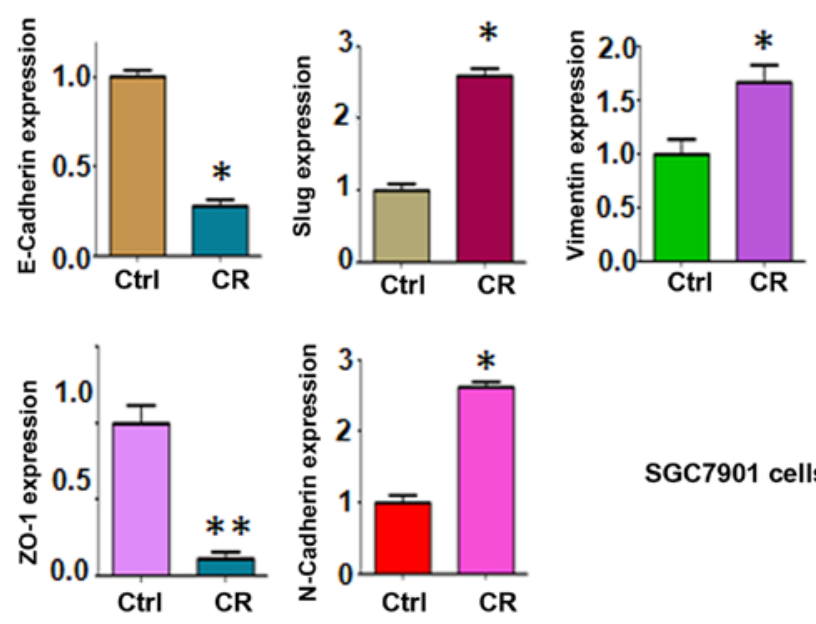

SGC7901 cells

Figure 3. Cisplatin-resistant (CR) cells have EMT marker changes. (A) Western blot analysis was used to detect the expression of E-cadherin, Snail, Slug, and Vimentin in MGC803 and MGC803 CR cells. Ctrl, control. (B) Quantitative results are illustrated for (A). ${ }^{*} \mathrm{P}<0.05,{ }^{* * *} \mathrm{P}<0.01$ vs. control. (C) Western blot analysis was performed to measure the expression of E-cadherin, Snail, Slug, and Vimentin in SGC7901 and SGC7901 CR cells. (D) Quantitative results are illustrated for $(\mathrm{C}) .{ }^{*} \mathrm{P}<0.05,{ }^{* *} \mathrm{P}<0.01$ vs. control.

EMT to MET. Our findings demonstrated that TAZ plays a critical role in chemoresistance and EMT in gastric cancer cells. Therefore, inhibition of TAZ could be a promising approach to overcome cisplatin resistance in gastric cancer.

Emerging evidence has demonstrated that chemoresistant cancer cells are associated with EMT (32). For example, gemcitabine-resistant pancreatic cancer cells displayed EMT via upregulation of Notch signaling pathway (33). Another study showed that gemcitabine-resistant hepatocellular carcinoma cells underwent EMT process through activation of PDGF-D pathway (24). Cisplatin resistance in gastric cancer cells is correlated with Her2 upregulation-induced EMT (34). CR cervical cancer cells exhibited EMT phenotype and overexpression of Sema4C (35). Consistent with these findings, our current study revealed that $\mathrm{CR}$ gastric cancer cells acquired EMT phenotype with decreased E-cadherin and increased expression of mesenchymal markers including Snail, Slug, Vimentin, and N-cadherin. Notably, EMT-type cells exhibited higher activities of migration and invasion. We confirmed that CR gastric cancer cells are associated with EMT.
Multiple studies have elucidated that some signaling pathways controlled cisplatin-induced chemoresistance. Sun et al found that NF- $\mathrm{BB}$ signaling played irreplaceable roles in cisplatin-induced chemoresistance and tumor progression in bladder cancer (36). Liu et al reported that cisplatin resistance is associated with increased motility and stem-like properties through upregulation of STAT3/Snail axis in tumor cells (37). One study showed that SET-mediated NDRG1 inhibition is involved in cisplatin resistance and EMT in human lung cancer cells (38). Another study demonstrated that Fbw7 upregulation promoted cisplatin cytotoxicity in non-small cell lung cancer cells (39). Wang et al reported that Akt/ $\beta$-catenin/Snail signaling pathway was associated with CR induced EMT in lung cancer cells (40). Downregulation of Par-4 conferred cisplatin resistance through PI3K (phosphoinositide 3-kinase)/Akt pathway-dependent EMT in pancreatic cancer cells (41). Connexin 43 has been found to reverse the resistance of lung adenocarcinoma cells to cisplatin through inhibition of EMT (42). Further study revealed that prolonged pemetrexed pretreatment enhanced 
A

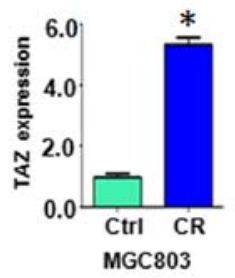

B

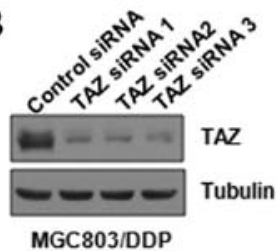

D MGC803/DDP

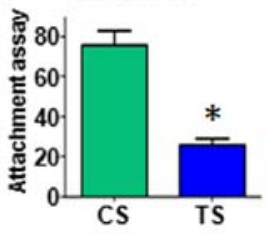

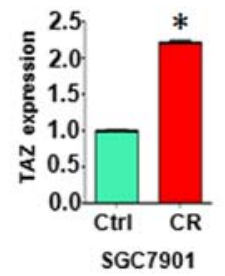

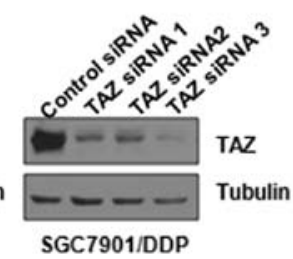

MGC803/DDP

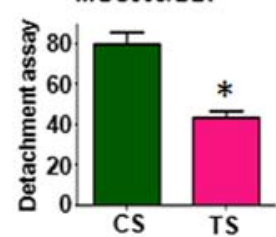

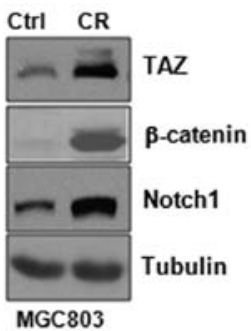

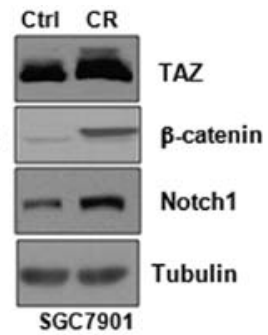

C

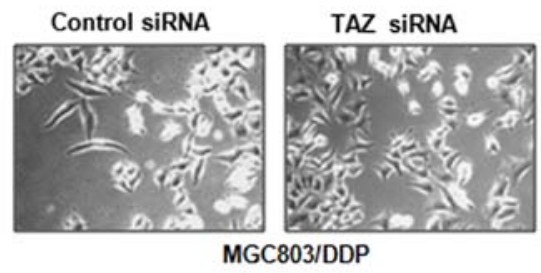

SGC7901/DDP

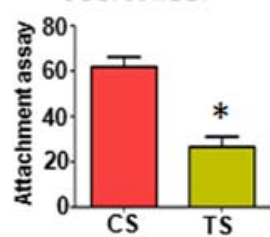

SGC7901/DDP

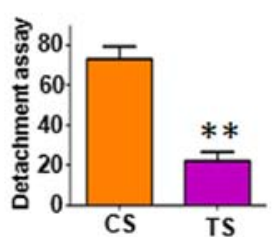

Figure 4. Cisplatin-resistant (CR) cells have high expression of TAZ. (A, left panel) Real-time RT-PCR assay was conducted to detect the expression of TAZ in parental and CR cells. "P<0.05 CR vs. control siRNA. (A, right panel) Western blot analysis was performed to detect the expression of TAZ in parental and CR cells. (B) Western blot analysis was performed to detect the efficacy of TAZ siRNA transfection. (C) Cell morphology was assessed by microscopy in CR cells transfected with TAZ siRNA. (D) Cell attachment and detachment assays were measured in CR cells transfected with TAZ siRNA. "P<0.05, ${ }^{* *} \mathrm{P}<0.01$ vs. control. CS, control siRNA; TS, TAZ siRNA.

A

MGC803/DDP
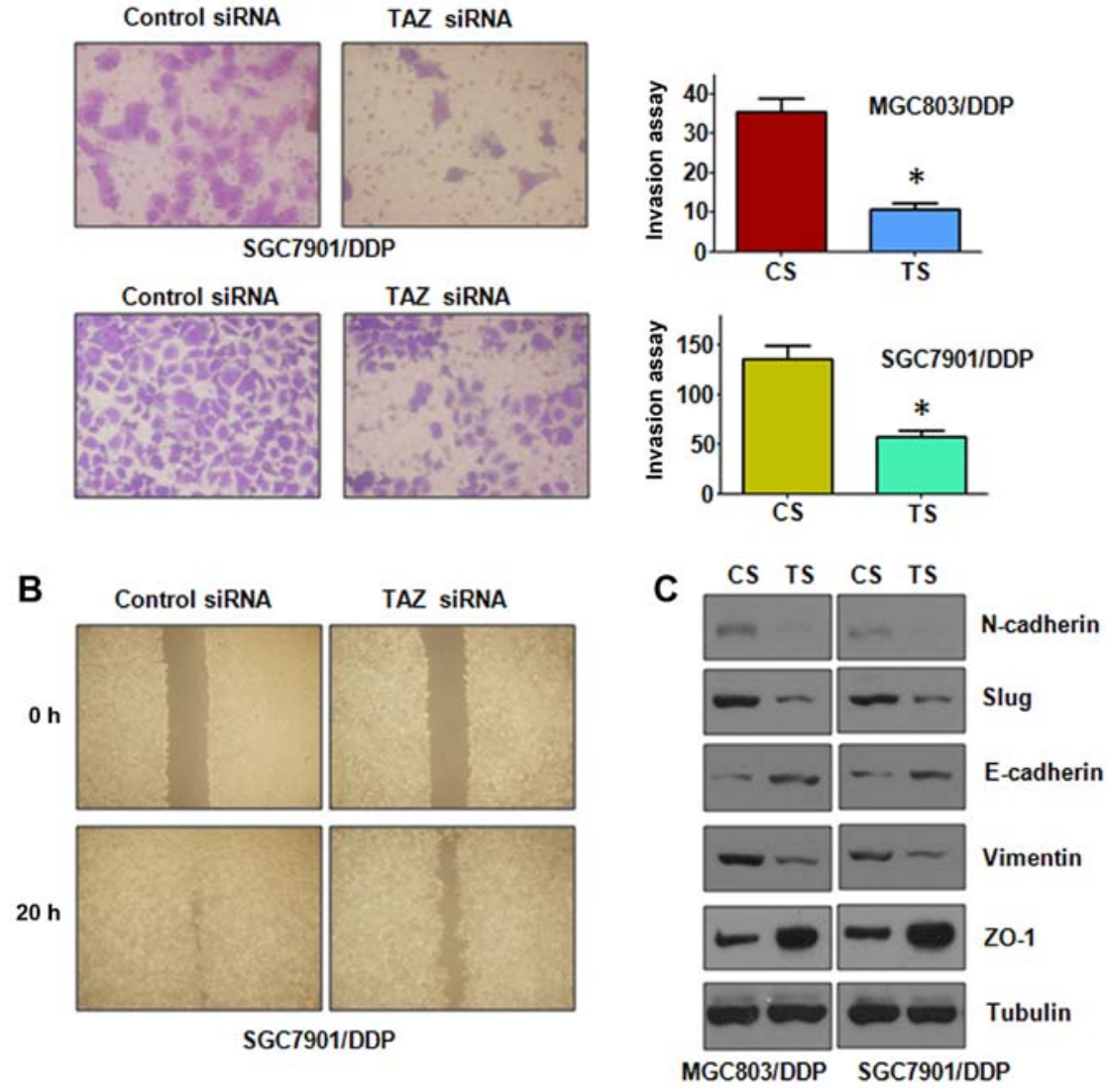

Figure 5. Depletion of TAZ inhibits motility and invasion and regulates expression of EMT markers in cisplatin-resistant (CR) cells. (A, top panel) Invasion assays were performed in CR cells transfected with TAZ siRNA. (A, bottom panel) Quantitative results are illustrated for the top panel. ${ }^{*} \mathrm{P}<0.05$ vs. control siRNA. (B) Wound healing assays were used to detect the motility in CR cells transfected with TAZ siRNA. (C) Western blot analysis was used to detect the expression of EMT markers in CR cells transfected with TAZ siRNA. CS, control siRNA; TS, TAZ siRNA. 
A

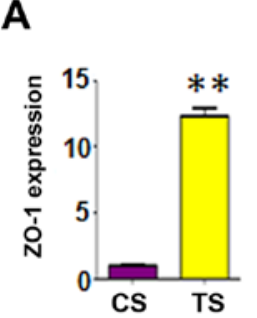

B

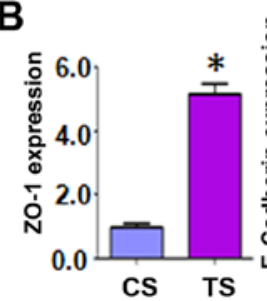

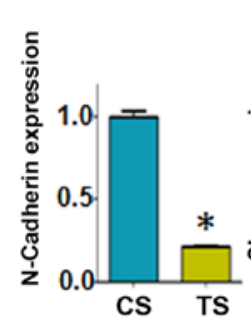
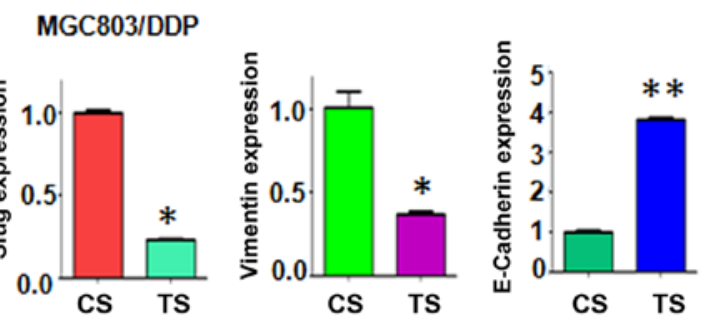

C
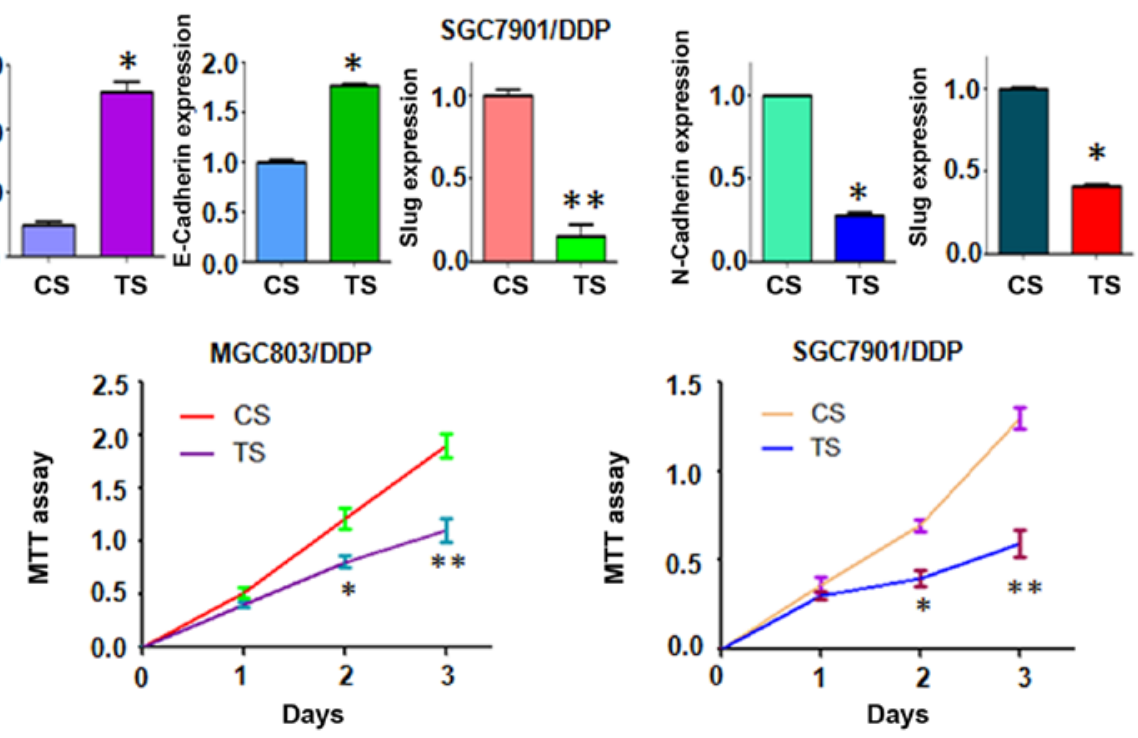

Figure 6. Depletion of TAZ regulates mRNA levels of EMT markers in cisplatin-resistant (CR) cells. (A and B) Real-time RT-PCR was performed to quantify mRNA expression of EMT markers in CR cells transfected with TAZ siRNA. CS, control siRNA; SS, TAZ siRNA. "P $<0.05$, "* P $<0.01$ compared with control siRNA. (C) MTT assay was performed in CR cells treated with TAZ siRNA. CS: control siRNA; TS: TAZ siRNA. "P<0.05, ${ }^{* *} \mathrm{P}<0.01$ compared with control siRNA.

persistence of cisplatin-induced DNA damage and eliminated tumor cells with EMT and cancer stem-like features in lung cancer cells (43). Our study identified that TAZ governed the CR-mediated EMT in gastric cancer cells. Further investigation is required to explore how TAZ controls CR-induced EMT in gastric cancer.

Accumulating evidence has demonstrated that miRNAs play an essential role in drug resistance and EMT in human cancers. For example, it has been observed that miR-20a induced cisplatin resistance via targeting CYLD in human gastric cancer cells (44). Zhao et al found that miR-181a suppressed autophagy and sensitized gastric cancer cells to cisplation (45). In addition, miR-26a enhanced the sensitivity of gastric cancer cells to cisplatin through targeting NRAS and E2F2 (46). Several miRNAs regulated the cisplatin resistance of human gastric cancer cells. Importantly, Chen et al reported that miR-206 regulated cisplatin resistance and EMT partly by targeting MET in human lung cancer cells (12). Wang et al found that miR-30a modulated EMT and cisplatin sensitivity in gastric cancer cells (13). Zuo et al identified that miR-141 inhibited tumor growth and metastasis via directly targeting TAZ in gastric cancer studies (47). These reports indicated that regulation of these miRNAs could be helpful to govern cisplatin-induced EMT.

In summary, our study validated that CR gastric cells acquired EMT in part due to overexpression of TAZ. In addition, consistent with other study $(26,48)$, TAZ was found to increase the expression of Notch-1 and $\beta$-catenin. Notch-1 and $\mathrm{Wnt} / \beta$-catenin have been characterized as the drivers to induce EMT $(33,49)$. Therefore, we believe that TAZ-induced EMT could be partly through upregulation of Notch-1 and $\beta$-catenin. Our results also indicated that downregulation of TAZ could be a useful strategy for restoring sensitivity to cisplatin. Indeed, one study has shown that knockdown of TAZ modified breast cancer cell sensitivity to EGFR inhibitors (50). In line with this, we found that depletion of TAZ sensitized CR cells to cisplatin treatment. Interestingly, one natural compound, curcumin, has been reported to inhibit the expression of TAZ in bladder cancer cells (51) and pancreatic cancer cells (48). Due to non-toxic nature of natural agents, inhibition of TAZ by these compounds could be a safe and effective approach for the prevention and the treatment of gastric cancer.

\section{Acknowledgements}

We thank our colleague for their critical reading.

\section{References}

1. Siegel RL, Miller KD and Jemal A: Cancer Statistics, 2017. CA Cancer J Clin 67: 7-30, 2017.

2. Chen W, Zheng R, Baade PD, Zhang S, Zeng H, Bray F, Jemal A, $\mathrm{Yu}$ XQ and He J: Cancer statistics in China, 2015. CA Cancer J Clin 66: 115-132, 2016.

3. Hayakawa Y, Sethi N, Sepulveda AR, Bass AJ and Wang TC: Oesophageal adenocarcinoma and gastric cancer: Should we mind the gap? Nat Rev Cancer 16: 305-318, 2016. 
4. Lordick F and Janjigian YY: Clinical impact of tumour biology in the management of gastroesophageal cancer. Nat Rev Clin Oncol 13: 348-360, 2016.

5. Wadhwa R, Song S, Lee JS, Yao Y, Wei Q and Ajani JA: Gastric cancer-molecular and clinical dimensions. Nat Rev Clin Oncol 10: 643-655, 2013

6. Petrelli F, Zaniboni A, Coinu A, Cabiddu M, Ghilardi M, Sgroi G and Barni S: Cisplatin or not in advanced gastric cancer: A systematic review and meta-analysis. PLoS One 8: e83022, 2013.

7. Orditura M, Galizia G, Sforza V, Gambardella V, Fabozzi A, Laterza MM, Andreozzi F, Ventriglia J, Savastano B, Mabilia A et al: Treatment of gastric cancer. World J Gastroenterol 20 : 1635-1649, 2014.

8. Chen DJ, Chen W, Jiang H, Yang H, Wang YC and Chen JH: Downregulation of DOCK1 sensitizes bladder cancer cells to cisplatin through preventing epithelial-mesenchymal transition. Drug Des Devel Ther 10: 2845-2853, 2016.

9. Du B and Shim JS: Targeting epithelial-mesenchymal transition (EMT) to overcome drug resistance in cancer. Molecules 21: 21, 2016.

10. De Craene B and Berx G: Regulatory networks defining EMT during cancer initiation and progression. Nat Rev Cancer 13: 97-110, 2013

11. Thiery JP, Acloque H, Huang RY and Nieto MA: Epithelialmesenchymal transitions in development and disease. Cell 139: 871-890, 2009.

12. Chen QY, Jiao DM, Wang J, Hu H, Tang X, Chen J, Mou H and Lu W: miR-206 regulates cisplatin resistance and EMT in human lung adenocarcinoma cells partly by targeting MET. Oncotarget 7: 24510-24526, 2016

13. Wang LL, Zhang XH, Zhang X and Chu JK: MiR-30a increases cisplatin sensitivity of gastric cancer cells through suppressing epithelial-to-mesenchymal transition (EMT). Eur Rev Med Pharmacol Sci 20: 1733-1739, 2016.

14. Haslehurst AM, Koti M, Dharsee M, Nuin P, Evans K, Geraci J, Childs T, Chen J, Li J, Weberpals J, et al: EMT transcription factors snail and slug directly contribute to cisplatin resistance in ovarian cancer. BMC Cancer 12: 91, 2012.

15. Kang W, Cheng AS, Yu J and To KF: Emerging role of Hippo pathway in gastric and other gastrointestinal cancers. World J Gastroenterol 22: 1279-1288, 2016.

16. Hong AW, Meng Z and Guan KL: The Hippo pathway in intestinal regeneration and disease. Nat Rev Gastroenterol Hepatol 13: 324-337, 2016.

17. Yue G, Sun X, Gimenez-Capitan A, Shen J, Yu L, Teixido C, Guan W, Rosell R, Liu B and Wei J: TAZ is highly expressed in gastric signet ring cell carcinoma. BioMed Res Int 2014: 393064, 2014.

18. Zhou GX, Li XY, Zhang Q, Zhao K, Zhang CP, Xue CH, Yang K and Tian ZB: Effects of the hippo signaling pathway in human gastric cancer. Asian Pac J Cancer Prev 14: 5199-5205, 2013.

19. Kim MH, Kim J, Hong H, Lee SH, Lee JK, Jung E and Kim J: Actin remodeling confers BRAF inhibitor resistance to melanoma cells through YAP/TAZ activation. EMBO J 35: 462-478, 2016.

20. Tian T, Li A, Lu H, Luo R, Zhang M and Li Z: TAZ promotes temozolomide resistance by upregulating MCL-1 in human glioma cells. Biochem Biophys Res Commun 463: 638-643, 2015

21. Lai D, Ho KC, Hao Y and Yang X: Taxol resistance in breast cancer cells is mediated by the hippo pathway component TAZ and its downstream transcriptional targets Cyr61 and CTGF. Cancer Res 71: 2728-2738, 2011.

22. Li Z, Wang Y, Zhu Y, Yuan C, Wang D, Zhang W, Qi B, Qiu J, Song X, Ye J, et al: The Hippo transducer TAZ promotes epithelial to mesenchymal transition and cancer stem cell maintenance in oral cancer. Mol Oncol 9: 1091-1105, 2015.

23. Yang Q, Huang J, Wu Q, Cai Y, Zhu L, Lu X, Chen S, Chen C and Wang Z: Acquisition of epithelial-mesenchymal transition is associated with Skp2 expression in paclitaxel-resistant breast cancer cells. Br J Cancer 110: 1958-1967, 2014.

24. Wu Q, Wang R, Yang Q, Hou X, Chen S, Hou Y, Chen C, Yang Y, Miele L, Sarkar FH, et al: Chemoresistance to gemcitabine in hepatoma cells induces epithelial-mesenchymal transition and involves activation of PDGF-D pathway. Oncotarget 4: 1999-2009, 2013.

25. Kong D, Wang Z, Sarkar SH, Li Y, Banerjee S, Saliganan A, Kim HR, Cher ML and Sarkar FH: Platelet-derived growth factor-D overexpression contributes to epithelial-mesenchymal transition of PC3 prostate cancer cells. Stem Cells 26: 1425-1435, 2008
26. Zhao Z, Zheng N, Wang L, Hou Y, Zhou X and Wang Z: Rottlerin exhibits antitumor activity via down-regulation of TAZ in non-small cell lung cancer. Oncotarget 8: 7827-7838, 2017.

27. Tan Y, Qin S, Hou X, Qian X, Xia J, Li Y, Wang R, Chen C, Yang Q, Miele L, et al: Proteomic-based analysis for identification of proteins involved in 5-fluorouracil resistance in hepatocellular carcinoma. Curr Pharm Des 20: 81-87, 2014.

28. Wang Z, Li Y, Ahmad A, Banerjee S, Azmi AS, Kong D and Sarkar FH: Pancreatic cancer: Understanding and overcoming chemoresistance. Nat Rev Gastroenterol Hepatol 8: 27-33, 2011.

29. Mallini P, Lennard T, Kirby J and Meeson A: Epithelial-tomesenchymal transition: What is the impact on breast cancer stem cells and drug resistance. Cancer Treat Rev 40: 341-348, 2014.

30. Xie D, Cui J, Xia T, Jia Z, Wang L, Wei W, Zhu A, Gao Y, Xie K and Quan M: Hippo transducer TAZ promotes epithelial mesenchymal transition and supports pancreatic cancer progression. Oncotarget 6: 35949-35963, 2015.

31. Xiao H, Jiang N, Zhou B, Liu Q and Du C: TAZ regulates cell proliferation and epithelial-mesenchymal transition of human hepatocellular carcinoma. Cancer Sci 106: 151-159, 2015.

32. Piskareva O, Harvey H, Nolan J, Conlon R, Alcock L, Buckley P, Dowling P, Henry M, O'Sullivan F, Bray I, et al: The development of cisplatin resistance in neuroblastoma is accompanied by epithelial to mesenchymal transition in vitro. Cancer Lett 364: $142-155,2015$.

33. Wang Z, Li Y, Kong D, Banerjee S, Ahmad A, Azmi AS, Ali S, Abbruzzese JL, Gallick GE and Sarkar FH: Acquisition of epithelial-mesenchymal transition phenotype of gemcitabineresistant pancreatic cancer cells is linked with activation of the notch signaling pathway. Cancer Res 69: 2400-2407, 2009.

34. Huang D, Duan H, Huang H, Tong X, Han Y, Ru G, Qu L, Shou C and Zhao Z: Cisplatin resistance in gastric cancer cells is associated with HER2 upregulation-induced epithelial-mesenchymal transition. Sci Rep 6: 20502, 2016.

35. Song J and Li Y: miR-25-3p reverses epithelial-mesenchymal transition via targeting Sema4C in cisplatin-resistance cervical cancer cells. Cancer Sci 108: 23-31, 2017.

36. Sun Y, Guan Z, Liang L, Cheng Y, Zhou J, Li J and Xu Y: NF-кB signaling plays irreplaceable roles in cisplatin-induced bladder cancer chemoresistance and tumor progression. Int J Oncol 48: 225-234, 2016

37. Liu WH, Chen MT, Wang ML, Lee YY, Chiou GY, Chien CS, Huang PI, Chen YW, Huang MC, Chiou SH, et al: Cisplatinselected resistance is associated with increased motility and stem-like properties via activation of STAT3/Snail axis in atypical teratoid/rhabdoid tumor cells. Oncotarget 6: 1750-1768, 2015.

38. Liu H, Gu Y, Yin J, Zheng G, Wang C, Zhang Z, Deng M, Liu J, Jia X and He Z: SET-mediated NDRG1 inhibition is involved in acquisition of epithelial-to-mesenchymal transition phenotype and cisplatin resistance in human lung cancer cell. Cell Signal 26: 2710-2720, 2014

39. Yu HG, Wei W, Xia LH, Han WL, Zhao P, Wu SJ, Li WD and Chen W: FBW7 upregulation enhances cisplatin cytotoxicity in non- small cell lung cancer cells. Asian Pac J Cancer Prev 14: 6321-6326, 2013

40. Wang H, Zhang G, Zhang H, Zhang F, Zhou B, Ning F, Wang HS, Cai SH and Du J: Acquisition of epithelial-mesenchymal transition phenotype and cancer stem cell-like properties in cisplatin-resistant lung cancer cells through AKT/ $\beta$-catenin/ Snail signaling pathway. Eur J Pharmacol 723: 156-166, 2014.

41. Tan J, You Y, Xu T, Yu P, Wu D, Deng H, Zhang Y and Bie P: Par-4 downregulation confers cisplatin resistance in pancreatic cancer cells via PI3K/Akt pathway-dependent EMT. Toxicol Lett 224: 7-15, 2014

42. Yu M, Zhang C, Li L, Dong S, Zhang N and Tong X: Cx43 reverses the resistance of A549 lung adenocarcinoma cells to cisplatin by inhibiting EMT. Oncol Rep 31: 2751-2758, 2014.

43. Tièche CC, Peng RW, Dorn P, Froment L, Schmid RA and Marti TM: Prolonged pemetrexed pretreatment augments persistence of cisplatin-induced DNA damage and eliminates resistant lung cancer stem-like cells associated with EMT. BMC Cancer 16: 125, 2016.

44. Zhu M, Zhou X, Du Y, Huang Z, Zhu J, Xu J, Cheng G, Shu Y, Liu P, Zhu W, et al: miR-20a induces cisplatin resistance of a human gastric cancer cell line via targeting CYLD. Mol Med Rep 14: 1742-1750, 2016.

45. Zhao J, Nie Y, Wang H and Lin Y: MiR-181a suppresses autophagy and sensitizes gastric cancer cells to cisplatin. Gene 576: 828-833, 2016. 
46. Wen L, Cheng F, Zhou Y and Yin C: MiR-26a enhances the sensitivity of gastric cancer cells to cisplatin by targeting NRAS and E2F2. Saudi J Gastroenterol 21: 313-319, 2015.

47. Zuo QF, Zhang R, Li BS, Zhao YL, Zhuang Y, Yu T, Gong L, Li S, Xiao B and Zou QM: MicroRNA-141 inhibits tumor growth and metastasis in gastric cancer by directly targeting transcriptional co-activator with PDZ-binding motif, TAZ. Cell Death Dis 6: e1623, 2015.

48. Zhou X, Su J, Feng S, Wang L, Yin X, Yan J and Wang Z: Antitumor activity of curcumin is involved in down-regulation of YAP/TAZ expression in pancreatic cancer cells. Oncotarget 7: 79076-79088, 2016.
49. Zhang J, Tian XJ and Xing J: Signal transduction pathways of EMT induced by TGF- $\beta$, SHH, and WNT and their crosstalks. J Clin Med 5: 5, 2016.

50. Guo L, Zheng J, Zhang J, Wang H, Shao G and Teng L: Knockdown of TAZ modifies triple-negative breast cancer cell sensitivity to EGFR inhibitors by regulating YAP expression. Oncol Rep 36: 729-736, 2016.

51. Gao Y, Shi Q, Xu S, Du C, Liang L, Wu K, Wang K, Wang X, Chang LS, He D, et al: Curcumin promotes KLF5 proteasome degradation through downregulating YAP/TAZ in bladder cancer cells. Int J Mol Sci 15: 15173-15187, 2014. 